\title{
New Integrated Framework for Video Based Moving Object Tracking
}

\author{
Md. Zahidul Islam, Chi-Min Oh, and Chil-Woo Lee \\ Chonnam National Univeristy, Gwang-ju, Korea
}

\begin{abstract}
In this paper, we depict a novel approach to improve the moving object tracking system with particle filter using shape similarity and color histogram matching by a new integrated framework. The shape similarity between a template and estimated regions in the video sequences can be measured by their normalized cross-correlation of distance transformation image map. Observation model of the particle filter is based on shape from distance transformed edge features with concurrent effect of color information. The target object to be tracked forms the reference color window and its histogram are calculated, which is used to compute the histogram distance while performing a deterministic search for matching window. For both shape and color matching reference template window is created instantly by selecting any object in a video scene and updated in every frame. Experimental results have been offered to show the effectiveness of the proposed method.
\end{abstract}

\section{Introduction}

Robust and reliable moving object tracking is one of the demanding and challenging task in computer vision problem such as visual surveillance, human computer interactions etc. Particle filters [1] provide a robust tracking framework as they are neither limited to linear systems nor require the noise to be Gaussian. The idea of a particle filter - to apply a recursive Bayesian filter based on sample sets - was independently proposed by several research groups [5], [11]. Our present work evolved from particle filter by improving observation model which is based on DT image map and color distribution.

In our previous work [12], a method has been suggested for a parametric video based object tracking using the particle filter. In this technique, we have solved the most two important problems which is faced basically for video based object tracking. The first one is segmentation difficulty which comes from illumination variation and cluttered background and second one is motion complexity of object itself. By using the feature of distinct corners and edges of the target object, the first problem is solved and for the second problem we have modeled the DOF of the object motion in 2D and 3D with some mathematics description. To observe the similarity of a hypothesis model in the image; the image and transformed feature model with a 3D transform matrix, we adopt chamfer matching method.

Tracking objects is performed in a sequence of video frames and it consists of two main stages: isolation of objects from background in each frames and association of 
objects in successive frames in to trace them. Object tracking in image processing is usually based on reference image of the object, or properties of the objects. To start tracking, generally the trackers need to be initialized by an outer component [15]. For example, a human operator can select an object of interest and let the tracking begin. Once initiated the tracking algorithms will conduct based on high correlations of the object motion, shape or appearance between consecutive video frames. But, unfortunately robust and efficient object tracking is still an open research issue. There are two central challenges; first one is visual measurements for tracking objects are not always dependable. To differentiate objects from background clutter, various image cues have been proposed such as object contour [9], edge [7], color distributions [8], [11] etc. And second one is tracking objects in nonlinear dynamic systems is not so easy. The Hidden Markov model provides a potential tool to solve the first difficulty. To address the second difficulty, particle filter (sequential Monte Carlo method) has been proposed extensively in various works [5], [6], and [11].

Shape and color both are very strong cue. The shape features, we use DT image map. In this present work, we are intended to use multi features for tracking. Only one cue sometimes is not enough for robust tracking and every individual cue has some individual limitations. We propose to use such a particle filter with DT image map and color-based image features as integrated framework for moving object tacking. A number of researchers used skin color but this is used only for hand and face tracking and it has some limitations like unique definition of skin color, shadows, occlusions and changing illuminations [5]. According to assumption in [10], the only moving objects in video scene are person. This postulation does not hold for many applications. But in this paper we try to make a general system for tracking any indoor outdoor object like person, car or hand. First we select any object form video scene manually, and then it is considered instantly as a reference image or template. Our system works in adaptive manner by updating template in every frame. The key mechanism of our developed observation model is based on similarity measures by normalized cross-correlation between DT image maps based template and tracking object in a video scene, and at the same time color histogram matching between reference image and target object. The more details we can find in overall proposed system model section.

The rest of the paper is organized as follows: section 2 describes related and existing work in this field. Section 3 introduces basic particle filter for object tracking. The proposed system models which unified distance transform, normalized crosscorrelation with simultaneous effect of color information by particle filter, are discussed in section 4 . Section 5 verify about the proposed system with some experimental results on various real video data in different environments. Conclusive remarks are addressed at the end of the paper in section 6.

\section{Particle Filter}

Tracking objects in video involves the modeling of non-linear and non-gaussian systems. In order to model accurately the underlying dynamics of a physical system, it is important to include elements of non-linearity and non-gaussianity in many application areas. Particle Filters can be used to achieve this. 
Particle filter is a sequential Monte Carlo methodology where the basic idea is the recursive computation of relevant probability distributions using the concepts of importance sampling and approximation of probability distributions with discrete random measures. The fundamental idea of Particle filter approximates the filtered posterior (next) distribution (density) by a set of random particles (sampling) with associated weights. It weights particles based on a likelihood score and then propagates these particles according to a motion model. Particle filtering assumes a Markov Model for system state estimation. Markov model states that past and future states are conditionally independent of a given current state. Thus, observations are dependent only on current state.

\subsection{Mathematical Description}

Particle filter consists of essentailly two steps: prediction and update. Given all available observations $y_{1: t-1}=\left\{y_{1}, \ldots, y_{t-1}\right\}$ up to time $t-1$, the prediction stage uses the probabilistic system transition model $p\left(x_{t} \mid x_{t-1}\right)$ to predict the posterior at time $\mathrm{t}$ as

$$
p\left(x_{t} \mid y_{t-1}\right)=\int p\left(x_{t} \mid x_{t-1}\right) p\left(x_{t-1} \mid y_{1: t-1}\right) d x_{t-1}
$$

At time $t$, the observation $y_{t}$ is available, the state can be updated using Bay's rule

$$
p\left(x_{t} \mid y_{1: t}\right)=\frac{p\left(y_{t} \mid x_{t}\right) p\left(x_{t} \mid y_{1: t-1}\right)}{p\left(y_{t} \mid y_{1: t-1}\right)}
$$

where $p\left(y_{t} \mid x_{t}\right)$ is described by the observation equation.

In the particle filter, the posterior $p\left(x_{t} \mid y_{1: t}\right)$ is approximated by a finite set of $N$ samples $\left\{x_{t}^{i}\right\}_{i=1, \cdots, N}$ with importance weights $w_{t}^{i}$. The candidate samples $\tilde{x}_{t}^{i}$ are drawn from an importance distribution $q\left(x_{t} \mid x_{1: t-1}, y_{1: t}\right)$ and the weight of the samples are -

$$
w_{t}^{i}=w_{t-1}^{i} \frac{p\left(y_{t} \mid \tilde{x}_{t}^{i}\right) p\left(\tilde{x}_{t}^{i}\right) \mid x_{t-1}^{i}}{q\left(\tilde{x}_{t} \mid x_{1: t-1}, y_{1: t}\right)}
$$

The samples are resampled to generate an unweighted particle set according to their importance weights to avoid degeneracy. In the case of the bootstrap filter [13], $q\left(x_{t} \mid x_{1: t-1}, y_{1: t}\right)=p\left(x_{t} \mid x_{t-1}\right)$ and the weights become the observation likeli$\operatorname{hood} p\left(y_{t} \mid x_{t}\right)$.

\subsection{Simple Mathematical Model of Our Proposed System}

For implementation of particle filter we need the following mathematical model:

1. Transition model / state motion model $P\left(x_{t} \mid x_{t-1}\right)$ : this specifies how objects move between frames.

2. Observation model $p\left(y_{t} \mid x_{t}\right)$ : this specifies the likelihood of an object being in a specific state (i.e. at the specific location). 
3. Initial state Est(1) / prior distribution model $p\left(x_{0}\right)$ : describes initial distribution of object states.

\section{Parametric 2D Model}

In our preceding work [12], we somehow did the same basic principle for video based object tracking like as our present work. In [12], we suggested a model based object tracking using particle filter with chamfer matching. In this paper, we model selected object features as color distribution and distance transform template with non Gaussian object movement and this algorithm is based on non geometric model which is little bit heavy and limited to simple model like scaling and rotating transform of object models.

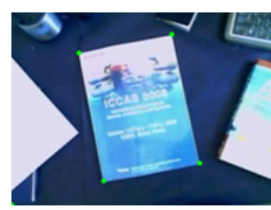

(1)

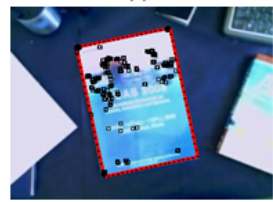

(3)

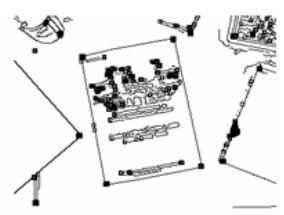

(2)

\section{Paramete}

Position : $(X, Y)=(112,204)$

Scale: 0.53885323

Width : 113.15918

Height : 153.73354

Z-Rotation Theta : -0.053047139

(4)

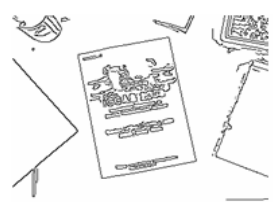

(1)

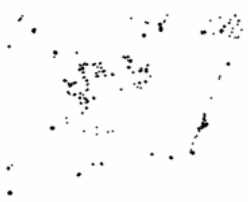

(3)

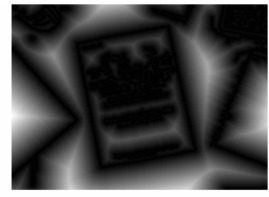

(2)

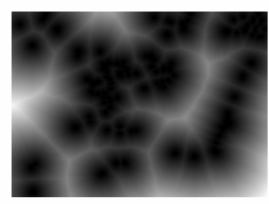

(4)

Fig. 1. Instance model (1) Four angular points (2) Corner features (3) Edge and corner features (4) Model information

Fig. 2. Feature and Distance Transform images (1) Canny Edge (2) Distance transform of Edge (3) Fast corner (4) Distance Transform of Corner

Our presented system is specially suited for a non geometric object which is far from camera position. As an ideal object model, we select A4 size book which has 4 angular points. By using these points, we select the model instance and the image transform factors are calculated as shown in Fig. 1. Chamfer matching is one of observation methods for verifying geometric edge model similarity in distance transform image. It observes how the feature points of hypothesis model are matched to distance transform of edge image. It uses feature points like edge and corner point from object model. Figure 2 shows feature images and distance transform images.

\section{Proposed Integrated Framework}

The task of robust tracking demands a robust observation model. Both shape and color are very important features to distinguish target object from background image. We integrate these two cues to develop our observation model. 


\subsection{Observation Model}

Basically the observation model is used to measure the observation likelihood of the samples and this is an important concern for object tracking. In the last few years, many observation models have been developed for particle filter tracking. In [5], a contour based appearance template is chosen to model the target. The tracker based on a contour template gives an accurate description of the targets but poorly in clutter, non rigid object and is generally time consuming. Also, the initialization is not easy and tricky in this method. On the other hand, the color based trackers are faster and more robust against contour based tracker. In this case, the color histogram is typically used to model the targets to combat the partial occlusion, and non-rigidity. The draw back of the color histogram is that spatial layout is ignored, and the trackers on it are easily confused by a background with similar colors. So, the combination of the two features provides better performance minimizing all the difficulties for any general tracking system.

The cross correlation based template matching is motivated by the distance measure (squared Euclidean distance). There are several disadvantages using this approach, for example, if the image energy varies with position, matching can fail. Moreover, it is not invariant to changes in image amplitude such as those caused by changing lighting conditions across the image sequences. The correlation coefficient overcomes these difficulties by normalizing the image and feature vectors to unit lengths, yielding a cosine-like correlation coefficient. So we are intended in our present work for DT image map based matching with normalized cross-correlation to develop observation model to make more robust particle filter based tracker. We try to take both advantages from DT image map and normalized cross correlation regarding to develop our particle filter based tracking system with color information.

\subsection{Initialization}

In our present case, for shape information, we propose normalized cross-correlation (NCC) based DT image matching. And for color information we use, simple HSV histogram based model. For our proposed tracking method we need to initialize the system, and this initialization working block diagram is shown in Fig. 3.

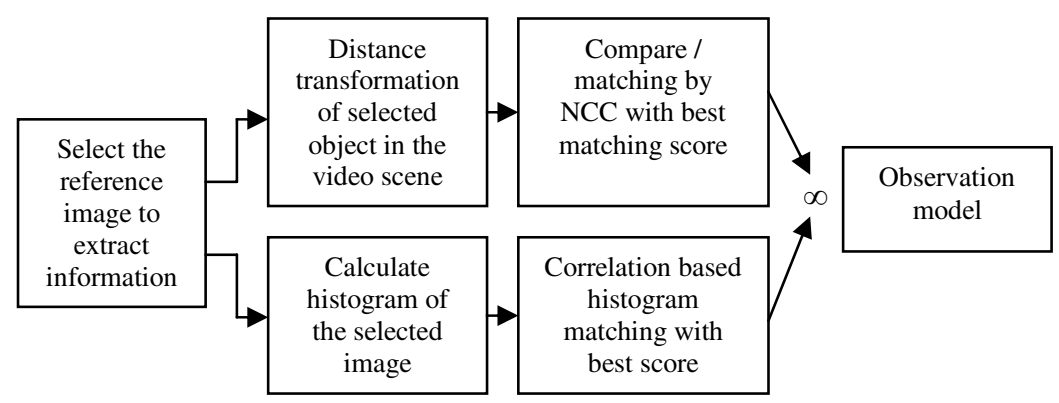

Fig. 3. Initialization steps. Whenever we select our reference image by a rectangle, then these primary actions starts to develop our observation model. 
DT image matching by NCC involves correlating the reference image with the distance transformed scene and determining the locations where the mismatch is blow a certain user defined threshold. The cross-correlation of template $t(x, y)$ with a subimage $f(x, y)$ is given by the following equation

$$
N_{f, t}=\sum_{x, y} \frac{(f(x, y)-\bar{f})(t(x, y)-\bar{t})}{\sigma_{f} \sigma_{t}}
$$

\subsection{Distance Transform (DT)}

The typical matching with DT [3] involves two binary images; a segmented template and a segmented image that we call feature template and feature image. It calculates the distance to closest zero pixel for all non-zero pixels of source image. To compute the distance transformation we use the function based on algorithm described in [2]. To formalize the idea of DT matching similar to chamfer matching [4], the shape of an object is represented by a set of point. The image map is represented as a set of feature points. Chamfer system basically depend on distance transform. Chamfer matching is a technique for finding the best fit of edge points from two different images, minimizing a generalized distance between them. A distance transform (DT) converts a binary image consisting of feature and non-feature pixels into an image where each pixel value denotes the distance to the nearest feature pixel. Distance image gives the distance to the nearest edge at every pixel in the image and it is calculated only once for each frame. In our present work we always update our reference template in every frame. As a result, it is more robust for matching in each changing of tracked object.

DT based matching has several advantages such as, in order to be tolerant to small shape variations, any similarity function between two shapes should vary smoothly when the feature point locations change by small amounts. By DT based system, we can match it very smoothly and robustly by means of normalized cross-correlation which is discussed in next section.

\subsection{Color Distribution Model}

We want to apply such a particle filter in color based context with integration with DT image map information. To achieve robustness against non-rigidity, rotation and partial occlusion we focus on color distributions as target models. These are represented by HSV based histogram of image. Color based probabilistic tracking rely on the deterministic search of a window, whose color content matches a reference histogram color model using principle of color histogram distance. We employ a function which compares two dense histograms using correlation method. If $\mathrm{H}_{1}$ denotes the first reference histogram and $\mathrm{H}_{2}$ denotes the target histogram then the correlation function is given by following equation 


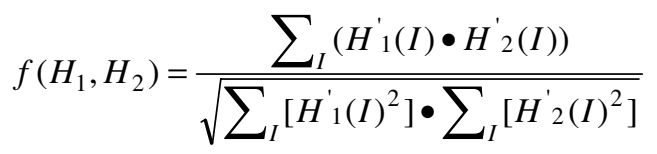

where

$$
\begin{aligned}
& H^{\prime}{ }_{k}(I)=H_{k}(I)-1 / N \bullet \sum_{j} H_{k}(J) \\
& (N=\text { number of histograms bins })
\end{aligned}
$$

\subsection{Particle Filter Based Implementation}

In our proposed system, we integrate DT based image matching by NCC and image histogram with particle filter for robust object tracking. In this particle filter based implementation we flow some steps, which are given below:

State space - we have modeled the states, as its location in each frame of the video. The state space is represented in the spatial domain as $\mathrm{X}=(\mathrm{x}, \mathrm{y})$. We have initialized state space for the first frame manually by selecting the object of interest in the video scene by rectangle.

System dynamics - a second-order auto regressive dynamics is chosen on the parameters used to represent our state space i.e. $(x, y)$. The dynamics is given as: $X_{t+1}=A x_{t}+B x_{t-1}$. Matrices $A$ and $B$ could be learned from a set of sequences where correct tracks have been obtained.

Observation $\mathbf{y}_{\mathbf{t}}$ - the observation $y_{t}$ is proportional to the NCC matching score between reference image and target image of the predicted location in the frame with time combine with the histogram best matching score. The strategy to take the best effect from combination of these of two features matching, we flow the following technical steps:

1. Score from DT image based shape matching by NCC.

2. Score from color histogram correlation based matching.

3. Normalized the both matching score.

4. Final score $=\alpha \times$ shape score $+\beta \times$ color score, where $(\alpha+\beta)=1$.

5. Search the particle with the best score.

According to this algorithm if color is dominant, then we increase $\beta$ and if shape is dominant, then we increase $\alpha$. We also can decide $\alpha$ and $\beta$ automatically. So, our present system developed the observation model which is based on the following equation -

$$
y_{t} \infty N C C\left(q, q_{x}\right)+\operatorname{Dist}\left(q, q_{x}\right)
$$

where $N C C=$ Normalized cross-correlation based matching score and Dist $=$ Histogram Distance score, $q$ and $q_{x}$ are reference image and target image respectively.

\section{Experiments and Results}

Our proposed system is suited in several environments for tracking any moving single objects. The combined effect of multiple features that is DT image map and color make 

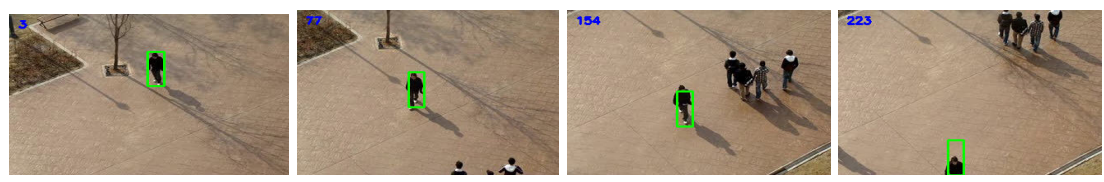

Fig. 4. First resulting sequences shows the single moving human tracking
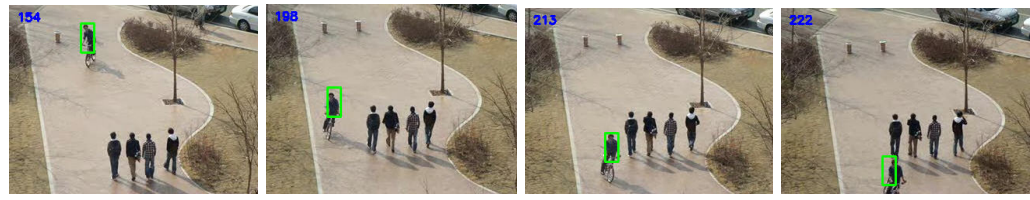

Fig. 5. Second resulting sequences of fast dynamic moving person with bicycle
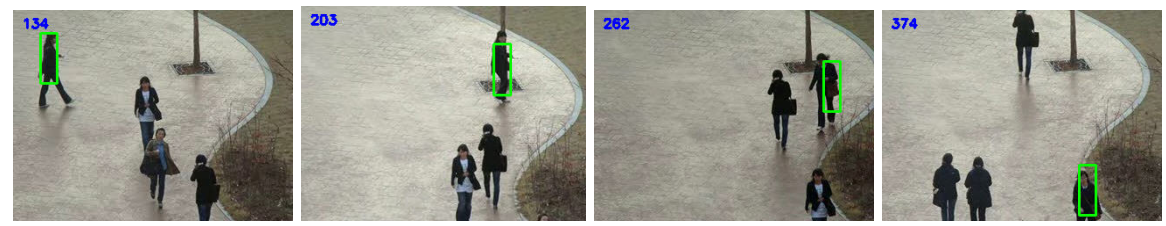

Fig. 6. Third resulting sequences of a dynamic moving person

our system more robust and reliable against some well known tacking problems. In this part we will point up some experimental results on several real-world video sequences captured by pan/tilt/zoom video camera in outdoor environment from the top of our department building. The captured sequences simulate various tracking conditions including quick movement, shape deformation, background clutter, appearance changes, camera pan/tilt/zoom, and partial occlusion. For all testing sequences, we use the same algorithm configuration.

In our first experiment result sequences shows the tracking of single moving person and after some time being this moving person passes away by a group of moving person. These are shown in Fig. 4. For each experiment, initialization is done by manually which make reference image instantly by selecting the region of interest (ROI) and we use 100 number particles. Our all resulting image sequences are presented here from left to right, the top left corner number shows the frame number.

The second resulting sequences as shown in Fig. 5 show some quick movement of a person with his cycle and randomly he changes his body appearance with cycle. As shown in Fig. 6 the third resulting video sequences the effectiveness of our proposed system as the moving person randomly changes her movement in very dynamic way.

This tracking algorithm is implemented in $\mathrm{C}++$ and OpenCV library on Windows XP platform with standard Pentium 4 (with 1.5 GB RAM) machine. Consequently, it can be concluded from all experimental results, that our proposed system can be very efficient general system as instant DT image and color histogram based object tracker. These all results show the algorithm performance under diverse scenarios. To illustrate the distribution of sample test, Fig. 7 shows the samples distribution considering 


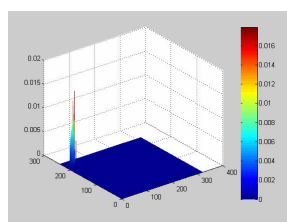

Frame \# 134

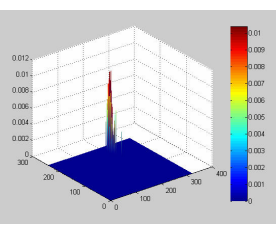

Frame \# 203

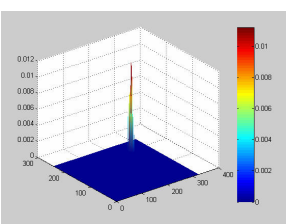

Frame \# 262

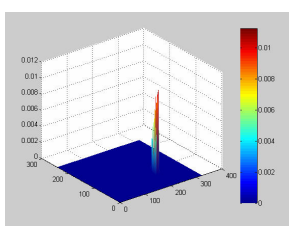

Frame \# 374

Fig. 7. Probability density functions of the corresponding moving person as shown in Fig. 5

the best matching score. This probability density function (pdf) represents the best match to the target object. These distribution results are taken from the same frame as represented in Fig. 6 respectively.

\section{Conclusion}

Our proposed tracking method successfully ensure some tracking conditions such as robustness and invariance of shape, non-rigidity due to its color distribution and DT image map similarity based observation model of particle filter. We take in to account for both color and shape features concurrently. Only color cues sometimes are not reliable for illumination changes, shadow and intensity changes. So, we are projected to add more cue like DT image map based shape which is invariant with some deformations of object like rotation, scaling etc. The observation model is updated by the best matching score in every frame. Initialization is necessary to start the tracking process and it is important that the tracking modules should be initialized effortlessly. The system has been tested on a variety of video data and very satisfactory results have been obtained. This work motivated us to do 3D information based object tracking as our future work.

Acknowledgements. This research work was supported by MIC \& IT leading R\&D support project [2006-S-028-01].

\section{References}

1. Djuric, P.M., Kotecha, J.H., Zhang, J., Huang, Y., Ghirmai, T., Bugallo, M.F., Miguez, J.: Particle Filtering. IEEE Signal Processing Magazine, 19-38 (2003)

2. Felzenszwalb, P.F., Huttenlocher, D.P.: Distance transforms of sampled functions. Cornell Computing and Information Science, TR2004 - 1963 (2004)

3. Gavrila, D.M.: Multi-feature Hierarchical Template Matching Using Distance Transforms. In: IEEE ICPR, Brisbane, Australia (1998)

4. Gunilla, B.: Hierarchical Chamfer Matching: A Parametric Edge Matching Algorithm. IEEE Transactions on Pattern Analysis and Machine Intelligence 10(6) (1988)

5. Israd, M., Blake, A.: CONDENSATION - conditional density propagation for visual tracking. International Journal of Computer Vision 29(1), 893-908 (1998)

6. Israd, M., Blake, A.: A mixed-state condensation tracker with automatic model-switching. In: International Conference on Computer Vision, pp. 107-112 (1998) 
7. Krishna, V.T., Kamesh, R.N.: Object tracking in video using particle filtering. In: IEEE International Conference on Acoustics, Speech, and Signal Processing, vol. 2, pp. 657-660 (2005)

8. Lehuger, A., Lechat, P., Perez, P.: An adaptive mixture color model for robust visual tracking. In: IEEE International Conference on Image Processing, pp. 573-576 (2006)

9. Li, P., Zhang, T., Pece, E.C.: Visual contour tracking based on particle filters. Image Vision Computing 21(1), 111-123 (2003)

10. Lu, W., Tan, Y.P.: A color histogram based people tracking system. In: ISCAS, vol. 2, pp. 137-140 (2001)

11. Nummiaro, K., Koller-Meier, E., Gool, L.V.: A color-based particle filter. In: First International Workshop on Generative-Model-Based Vision, pp. 53-60 (2002)

12. Chimin, O., Islam, M.Z., Lee, C.W.: Two Dimensional edge and corner model based object tracking using particle filter. In: 15th Japan-Korea Joint Workshop on Frontiers of Computer Vision, pp. 223-228 (2008)

13. Sanjeev, A.M., Simon, M., Neil, G., Tim, C.: A tutorial on particle filters for online nonlinear/non-gaussian Bayesian tracking. IEEE Transactions on Signal Processing 50(2), 174-188 (2002)

14. Stenger, B.D.R.: Model-Based Hand Tracking Using A Hierarchical Baysian Filter. PhD Thesis, University of Cambridge (2004)

15. Yunqiang, C., Yong, R.: Real time object tracking in video sequences. Signals and Communications Technologies, Interactive Video. Part II, 67-88 (2006)

16. Zhao, F., Huang, Q., Gao, W.: Image Matching by Normalized Cross-Correlation. In: IEEE International Conference on Acoustics, Speech and Signal Processing, vol. 2, pp. II14-II-19 (2006) 